\title{
An investigation into the role of coping in preventing depression associated with perfectionism in preadolescent children
}

\author{
Silja M. Dry ${ }^{*}$, Robert Thomas Kane and Rosanna M. Rooney \\ Curtin University, Perth, WA, Australia
}

The relationships between self-oriented and socially prescribed perfectionism (SPP) and maladaptive and adaptive coping strategies and their collective impact on depression symptoms were examined in the context of a randomized controlled universal trial of the Aussie Optimism Positive Thinking Skills Program. Five hundred and forty-one children aged 8-12 completed a battery of self-reports, of which responses for measures of depression symptoms, perfectionism, and coping strategies were examined for the purposes of this study. Structural equation modeling tested whether coping mediated

OPEN ACCESS

Edited by:

Frederick Robert Carrick,

Carrick Institute, USA

Reviewed by:

Linda Mullin Elkins,

Life University, USA

Michael Van Doren Johnston,

Kennedy Krieger Institute, USA

*Correspondence:

Silja M. Dry,

Curtin University, Perth, WA, Australia silja.dry@postgrad.curtin.edu.au

Specialty section: This article was submitted to Child Health and Human Development, a section of the journal

Frontiers in Public Health

Received: 03 June 2015

Accepted: 21 July 2015

Published: 07 August 2015

Citation:

Dry SM, Kane RT and Rooney RM (2015) An investigation into the role of coping in preventing depression associated with perfectionism in preadolescent children.

Front. Public Health 3:190. doi: 10.3389/fpubh.2015.00190 the effects of perfectionism on depression. Results indicated that SPP had both a direct and an indirect relationship with depression symptoms through a moderate association with maladaptive coping. Implications for prevention of depression were discussed and recommendations for future research were proposed.

Keywords: middle childhood, perfectionism, coping, depression

\section{Introduction}

Perfectionism or striving for flawlessness is considered an adaptive trait in high achievers and elite athletes as it drives the individual to succeed and as a result contributes positively to mental well-being by improving life satisfaction and self-esteem (1-4). However, as a growing body of research has established, perfectionism may be maladaptive when motivated through a fear of failure, compelling individuals to engage in perfectionistic behavior to avoid failure $(5,6)$. Individuals with maladaptive perfectionism traits tend to engage in critical self-evaluation against a rigid set of unrealistically high expectations, leading to dichotomous thinking, rumination over mistakes, procrastination, increased stress, dysphoria, and performance dissatisfaction $(7,8)$.

If follows that maladaptive perfectionism may contribute to the development and maintenance of depression, a clinical disorder that may manifest during adolescence (9-11). As perfectionism traits may emerge at an early age in childhood, research into the interactions with pre-clinical symptoms of depression in children could potentially inform preventive strategies (12).

In examining the relationship between maladaptive perfectionism and depression in children, researchers commonly utilize the Child and Adolescent Perfectionism Scale (CAPS) developed by Flett colleagues, an adaptation of the Multidimensional Perfectionism Scale (MPS) devised to assess intra and interpersonal dimensions of perfectionism in adult populations (13). The measure like its adult counterpart differentiates between self-oriented perfectionism (SOP), the striving for, and critical self-evaluation against self-imposed excessively high standards; and socially prescribed perfectionism (SPP), the striving for, and critical self-evaluation against, the perceived expectation 
of others (Flett et al., Unpublished) ${ }^{1}$. However, the CAPS omits the "other oriented" perfectionism dimension, which refers to perfectionism being imposed onto others as Flett et al. (Unpublished) ${ }^{1}$ found no developmental evidence to support this factor. Studies with adolescents demonstrated robust associations between symptoms of depression and both SOP and SPP in the context [e.g., Ref. (14-17)], generally replicating findings in adults [see Ref. (9)], while research in younger samples has been limited and with mixed results. Nobel et al. (18) observed that only SOP was significantly correlated with depression in 8 - to 11-year-old children identified at-risk for depression or anxiety. This was inconsistent with earlier findings by Huggins et al. (19) who observed that only SPP was associated with a clinical diagnosis of depression in a group of indicated preadolescents (10-11 years). This discrepancy may be due to a potential floor effect in the Nobel et al. study with mean SPP scores almost 2 SDs lower than those reported by Huggins et al. Furthermore, Nobel et al. applied a reduced version of the CAPS, potentially limiting detection of a significant interaction between SPP and depression, as well as its comparability with other studies. Additional research with a larger sample of preadolescents is therefore needed to clarify the nature of association between depression and perfectionism in children.

In examining relationships between perfectionism and depression in adults, researchers have observed that individuals with high levels of perfectionism tend to apply ineffective coping strategies to manage stressful events $(20,21)$. Coping strategies are considered effective and adaptive when behavioral and cognitive processes include active emotion regulation, constructive thinking, and problem solving techniques to reduce stress (22); and they are considered ineffective or maladaptive when comprising avoidant cognitive and behavioral strategies, which tend to be associated with poorer psychological outcomes $(23,24)$. Researchers have found that coping is often context driven and depends on the initial appraisal of the situation by the child and their coping repertoire, which becomes more diversified and sophisticated with development $(25,26)$.

Research has established that adults with high levels of maladaptive perfectionism, specifically SPP, are more likely to apply maladaptive coping (MCOP) and less likely to apply adaptive coping $(21,27)$. Research on a sample of maltreated adolescents has indicated similar patterns of associations with Flett et al. (28) observing that SOP was correlated with problem solving (an adaptive coping strategy), while SPP was negatively correlated to problem solving and positively related to avoidant (maladaptive) coping.

Studies utilizing structural equation modeling (SEM) have demonstrated that MCOP either fully or partially mediate the relationship between perfectionism and depression in adults [e.g., Ref. (29-31)]. However, no known published research has examined the mediating role of coping on the depression-perfectionism relationships in children.

Exposing the interactions of MCOP, perfectionism, and depression may have implications for the development of effective treatment and prevention of depressive disorders, particularly

\footnotetext{
${ }^{1}$ Flett GL, Hewitt PL, Boucher DJ, Davidson LA, Munro Y. The Child-Adolescent Perfectionism Scale: Development, Validation, and Association with Adjustment (2000), (Unpublished).
}

given Blatt and colleagues' findings in a landmark study [see Ref. (32)] that perfectionism can limit the effectiveness of otherwise efficacious treatment for depression in adults. Interventions addressing depressive symptoms in adults with high levels of perfectionism have only been efficacious when directly targeting perfectionistic behaviors and cognitions [e.g., Ref. (33-35)].

Although there are no known evidenced-based treatments for children that specifically target perfectionism, preliminary research indicates that CBT-based interventions aimed at preventing and reducing depression symptoms may also influence perfectionism. Essau et al. (36) reported significant improvements in both SOP and SPP as well as coping and depression symptoms as a result of a randomized controlled trial of the manualized FRIENDS program administered to a large community sample of 9- to 12-year-old children. A randomized controlled study by Nobel et al. (18) in children with subclinical levels of anxiety and depression also observed reductions in depression and SOP. As there were no significant differences between treatment and control groups at post-test, conclusions regarding treatment efficacy were limited. A study by Dry (37) found positive changes over time in depression levels of 8- to 11-year-old children with high levels of perfectionism in an open trial of the CBT-based Aussie Optimism Positive Thinking Skills program. However, the absence of a control group limited inferences that could be made about treatment efficacy.

The purpose of this study was to address specific limitations of the aforementioned research and examine the interrelationships between coping strategies, perfectionism, and prodromal depression symptoms in the context of a preventative treatment for pre adolescents. It was specifically hypothesized that:

H1: Pre-test perfectionism (SOP and SPP) would moderate the intervention effect on depression scores, such that children with high-perfectionism scores would benefit less from the intervention;

$\mathrm{H} 2$ : At pre-test, MCOP would partially mediate the relationship between perfectionism (SOP and SPP) and depression symptoms, such that individuals with high levels of perfectionism would score more highly on MCOP and depression symptoms. It was expected that this mediation relationship would be stronger for SPP. As researchers reported gender differences in rates of SOP and SPP (28), in coping strategies [e.g., Ref. (38)] and rates of depression symptoms (11), it was expected that gender would be a covariate; and

H3: Adaptive coping would partially mediate the therapeutic effect of the intervention on depressive symptoms.

\section{Materials and Methods}

\section{Participant Characteristics}

The sample comprised 541 children (49.5\% males, $50.5 \%$ females) aged $8-12$ years $(M=9.72, S D=1.06)$ from a predominantly Australian background (87.7\%). Children attended years 4 (56\%) and $5(44 \%)$ at 10 Catholic primary schools from lower to middle socioeconomic ranked suburbs within the Perth Metropolitan region, WA, Australia. Demographic information was derived from questionnaires completed by $77 \%$ of parents. 


\section{Sampling Procedures}

This study's sample was drawn from a larger longitudinal study, the 2012 Aussie Optimism Positive Thinking Project (AO-PTS). AO-PTS researchers randomly selected schools from a pool of 30 co-educational independent schools that satisfied a double stream per year minimum class size requirement and an absence of prior treatment programs. Schools were paired according to the socioeconomic status measure of the school's postcode and class size and then randomly assigned to either the intervention or control conditions. The study sample comprised the first five intervention and control groups for which pre- and post-test data collection had been completed at the time of analysis.

\section{Research Design}

A cluster randomized controlled trial was undertaken to investigate the relationships between a universal intervention program and pre and post measures of perfectionism, coping, and depression. The endogenous variables were children's depression symptoms, maladaptive, and adaptive coping strategies; the exogenous variable was children's pre-test perfectionism.

\section{Sample Size}

A priori power analysis using Kline (39) recommendations of a 20:1 sample size-to-parameter ratio was used for estimating the sample size for the Structural Equation models. Based on a maximum number of 13 parameters, the obtained sample size of 541 well exceeded the a priori determined sample size of 260 .

\section{Instruments}

\section{Intervention}

The Aussie Optimism: Positive Thinking Skills Program (40) is a CBT-based universal intervention aimed at preventing internalizing disorders in preadolescents. The manualized program was administered weekly to students over $1 \mathrm{~h}$ session for a period of 10 weeks by teachers specifically trained in the intervention. The 10 treatment modules included the following age appropriate content: positive activity scheduling; identifying feelings in self and others; situational impact on feelings; overcoming fear; becoming aware of thoughts and their connection to feelings; awareness of helpful and unhelpful thinking; challenging unhelpful thinking and adopting more positive thoughts, and consolidation of principles learned. A previous trial of the universally administered classroom-based program demonstrated reductions in depressive symptoms (41).

\section{Measures}

The following lists the self-rated measures relevant to this project administered as part of the larger AO-PTS assessment battery.

The Child and Adolescent Perfectionism Scale (Flett et al., Unpublished $)^{1}$ is a widely used 22 -item measure to assess children's self-oriented and SPP tendencies. The items have been worded to be suitable for children of a 3-year level reading age and are self-rated on a 5-point scale (Flett et al., Unpublished) ${ }^{1}$. Flett et al. (Unpublished) ${ }^{1}$ demonstrated sound reliability and test-retest reliabilities for the full scale rates and both subscales, and discriminant and concurrent validity. Cronbach's alpha rates in this sample were $\alpha=0.84$ for SPP and $\alpha=0.73$ for SOP, the latter being somewhat marginal for the purposes of analysis and considerably lower than that reported by Flett et al. $(\alpha=0.85)$.

The Children's Depression Inventory [CDI; (42)] is a 27-item scale frequently used in research and clinical applications. Children are required to select one of three statements that most closely reflect their dominant emotional state in the previous 2 weeks. Statements indicate absent, mild, or strong depression symptoms. Item No. 19 (evaluating suicide ideation) was removed from the questionnaire due to concerns raised by school principals (43). The abbreviated scale in this sample yielded a Cronbach's alpha reliability rate of $\alpha=0.89$. Kovacs (42) demonstrated discriminant and concurrent validity for the scale and reported sound test-retest reliability rates, ranging from $r=0.66-0.83$ at 2-4 weeks.

The Coping Scale for Children and Youth [CSCY; (44)] is a 29item 4-point scale in which children rate how frequently they use a particular coping behavior in response to a problem. The scale differentiates between adaptive coping styles of assistance seeking and cognitive behavioral problem solving, and MCOP styles of cognitive avoidance and behavioral avoidance. Essau et al. (36) reported an alpha reliability of $\alpha=0.84$. The current sample yielded Cronbach's alpha of 0.82 and 0.85 for the adaptive and maladaptive subscales, respectively. This instrument was reported to have sound test-retest reliability ranging from $r=0.73$ to 0.81 (44).

\section{Data Collection Procedure}

Ethics approval was obtained from both the Curtin University Human Ethics Committee and the Catholic Education Commission. Once school principals had agreed to participate, written informed consent was sought from parents and children. Trained experimenters, who were blind to the treatment mode, administered the battery of measures in accordance with the AO-PTS protocol manual to participating children in classroom groups during class time, reading aloud all questions to minimize differences in reading ability. Where possible a teacher was present at testing to ensure duty of care was maintained. Children were rewarded with a sticker and pencil or eraser for participating. After data entry, the AO-PTS research team identified children who scored highly on the CDI [above 17, Ref. (42)]. Parents were notified accordingly offered referrals for appropriate psychological support for their children.

Testing was conducted before and after the 10-week intervention, or in the case of the control group after an equivalent lapse of time. Data collection occurred between 17 September 2010 and 14 December 2010 for the intervention schools and between 3 August 2011 and 6 December 2012 for the control schools. The difference in timing was due to difficulties in recruiting control schools.

Records of attendance rates and treatment compliance were maintained by teachers but remained incomplete to date. Although data were analyzed on intent-to-treat basis, interpretation of treatment effects should be made with caution.

\section{Data Analysis}

Hypotheses were tested by means of SEM using LISREL v. 8.80 software (45). An advantage of SEM is the ability to input alpha reliabilities to reflect measurement error of measures used (46). 
Assumption and prerequisite testing for the SEM were undertaken using Statistical Package for the Social Sciences (SPSS) version 19.0 software.

\section{Results}

\section{Participant Flow}

Parental and student consent for participation was obtained for 650 (50.15\%) of 1,296 eligible students. Twenty-two students (11 in each condition) were absent at pre-test, while 43 students (16 in the intervention group, 27 in the control group) were absent at post-test. Consent was withdrawn for 21 students. Cases with more than $15 \%$ of missing values over a single variable amounted to 14 at pre-test and an additional 15 cases at post-test ( 1 of whom withdrew consent). After deleting cases of consent withdrawal, partial wave non-response and cases with excessive missingness, 541 cases were available for analysis.

\section{Screening and Missing Values}

Missing data extended to $6.4 \%$ of responses, with 101 out of 164 variables recording more than $5 \%$ missing values (maximum 9.7\%). Little's MCAR test was significant with $\chi^{2}(34,696$, $N=650)=36,460.88, p<0.001$, indicating that data were not missing completely at random. Prior to pairwise deletion, the 88 cases of partial or complete wave non-response were screened for elevations on the CDI (scores above 17). Fourteen children (six in the intervention and three in the control group) were identified at being at-risk for depression, thus deletion of these cases may have biased results.

However, cases of partial or full wave non-response needed to be deleted pairwise as the SEM is not robust to missingness (39). For the remaining 541 cases, where missingness was $<15 \%$ per measure, missing values were replaced using a maximum likelihood estimation, expectation maximization (EM), in SPSS (Version 19). While values were not missing at random, the true cause of missingness is usually only moderately correlated with outcomes and therefore replacing missing values with EM would be of minimal consequence to estimates $(47,48)$.

\section{Descriptive Statistics}

Table 1 shows the mean of total scores and SDs in addition to the range of scores for all outcome variables for both waves of testing according to test condition. The mean scores for depression symptoms in both groups, while higher in the control group, were well below the clinically significant cut off levels $(>17)$, which would be consistent with a healthy community sample. Despite low means scores, 54 children were identified as being at-risk for depression ( 24 in the intervention group and 30 in the control group) at pretest, while 50 children were identified at-risk for depression (27 in the intervention group and 23 in the control group) at posttest. Mean scores for SPP and SOP were comparable to those reported by Huggins et al. (19), Flett et al. (Unpublished) ${ }^{1}$, and Flett et al. (14).

\section{Assumption Testing}

The data were screened for the assumptions of univariate and multivariate normality, linearity, absence of multicollinearity and singularity of covariance, and symmetrical distribution of residuals (46). Violations to univariate normality were observed on CDI scores, which were positively skewed. A test of multivariate normality for continuous variables was significant with $\chi^{2}=280.06$, $p<0.001$. A violation to normality necessitated the computation of a Spearman correlation matrix for input into the structural equation model (49). Although multivariate outliers were detected for 12 cases, exceeding the critical Mahalanobis chi-square values $\left[\chi^{2}(16)=36.12, p<0.001\right]$, these were retained as correlation matrices were unaffected.

\section{Prerequisite Analyses}

As hypotheses $\mathrm{H} 1$ and $\mathrm{H} 3$ were examining relationships between variables in the context of an intervention effect, the presence of an intervention effect on outcome variables needed to be established. This was achieved with the use of the Generalized Linear Mixed Models (GLMM) procedure, which produces a multi-level mixed effects linear regression. This is the statistical method of choice for examining intervention effects in data that are multi-level in nature, has unequal group sizes (intervention

TABLE 1 | Scores on outcome measures for control and intervention groups at both waves of testing

\begin{tabular}{|c|c|c|c|c|c|c|}
\hline Measure & Mean (SD) & Range & $95 \% \mathrm{Cl}$ & Mean (SD) & Range & $95 \% \mathrm{Cl}$ \\
\hline & \multicolumn{3}{|c|}{ Pre-test } & \multicolumn{3}{|c|}{ Post-test } \\
\hline \multicolumn{7}{|c|}{ INTERVENTION } \\
\hline CDI & $7.06(6.40)$ & 0-33 & {$[6.35,7.77]$} & 6.18(6.92) & $0-40$ & {$[5.41,6.95]$} \\
\hline CSCY-A & $2.65(0.54)$ & $1.25-3.92$ & {$[2.58,2.71]$} & $2.62(0.53)$ & $1.08-3.92$ & {$[2.56,2.68]$} \\
\hline CSCY-M & $2.31(0.53)$ & $1.06-4.00$ & {$[2.25,2.36]$} & $2.30(0.57)$ & $1.00-4.00$ & {$[2.24,2.37]$} \\
\hline SPP & $27.24(7.68)$ & $10-50$ & {$[26.39,28.10]$} & 26.11 (8.09) & $10-50$ & {$[25.21,27.01]$} \\
\hline SOP & $37.94(6.60)$ & $12-60$ & {$[37.21,38.67]$} & $36.81(7.18)$ & $12-60$ & {$[36.01,37.61]$} \\
\hline \multicolumn{7}{|c|}{ CONTROL } \\
\hline CDI & 8.93 (8.09) & $0-40$ & {$[7.86,10.00]$} & 7.56 (7.39) & $0-46$ & {$[6.59,8.53]$} \\
\hline CSCY-A & $2.62(0.56)$ & $1.04-3.93$ & {$[2.55,2.70]$} & $2.55(0.61)$ & $1.00-4.00$ & {$[2.47,2.63]$} \\
\hline CSCY-M & $2.32(0.50)$ & $1.06-3.9$ & {$[2.25,2.35]$} & $2.20(0.51)$ & $1.00-3.76$ & {$[2.13,2.27]$} \\
\hline SPP & 27.06 (7.98) & $10-48$ & {$[26.01,28.10]$} & $25.47(8.63)$ & $9-50$ & {$[24.34,26.60]$} \\
\hline SOP & 38.38 (6.67) & $21-58$ & {$[37.50,39.25]$} & $37.10(7.56)$ & $12-60$ & {$[36.11,38.09]$} \\
\hline
\end{tabular}

Intervention group $A=314$, control group $N=227$.

$\mathrm{Cl}$, confidence interval; $\mathrm{CDI}$, child depression inventory; CSCY, coping scale for children and youth; $\mathrm{A}$, adaptive coping; $M$, maladaptive coping; SPP, socially prescribed perfectionism; SOP, self-oriented perfectionism.

Missing values were deleted list wise where missingness was $>15 \%$ per variable per case, other missing values were EM replaced. 
TABLE 2 | Fixed effects estimates for interactions with time and intervention mode.

\begin{tabular}{|c|c|c|c|}
\hline Source & $\boldsymbol{F}$ & Significance & Effect size $\eta^{2}$ \\
\hline \multicolumn{4}{|l|}{ CDI } \\
\hline Group & 4.90 & $0.01^{*}$ & $<0.00$ \\
\hline Time & 8.5 & $0.03^{\star}$ & 0.01 \\
\hline Group $\times$ time & 0.42 & $0.00^{\star \star}$ & $<0.00$ \\
\hline \multicolumn{4}{|l|}{ SPP } \\
\hline Group & 0.23 & 0.64 & \\
\hline Time & 29.41 & $0.00^{\star \star \star}$ & 0.03 \\
\hline Group $\times$ time & 0.81 & 0.37 & \\
\hline \multicolumn{4}{|l|}{ SOP } \\
\hline Group & 0.41 & 0.52 & \\
\hline Time & 16.16 & $0.00^{\star * *}$ & 0.01 \\
\hline Group $\times$ time & 0.08 & 0.78 & \\
\hline \multicolumn{4}{|c|}{ ADAPTIVE COPING } \\
\hline Group & 0.09 & 0.77 & \\
\hline Time & 4.96 & $0.03^{*}$ & $<0.00$ \\
\hline Group $\times$ time & 1.11 & 0.29 & \\
\hline \multicolumn{4}{|c|}{ MALADAPTIVE COPING } \\
\hline Group & 0.38 & 0.54 & \\
\hline Time & 5.54 & $0.02^{*}$ & $<0.00$ \\
\hline Group $\times$ time & 5.20 & $0.02^{*}$ & $<0.00$ \\
\hline
\end{tabular}

dfnumerator $=1$, dfnominator $=1,078 .{ }^{*} p<0.05 ;{ }^{* *} p<0.01 ;{ }^{* * *} p<0.001$.

and control), and in which outcome variables correlate with each other between tests (50). It also makes adjustments to address violations to normality. To take into account the nested nature of the data, participants, year, and schools were controlled for as random factors in the model. Fixed factors included one categorical fixed effect (group: intervention, control), and one ordinal fixed effect (time: pre, post).

Results of the GLMM, summarized in Table 2, demonstrate that there was a significant, albeit small, interaction with mean scores for all measures over time. While there was a significant interaction of treatment mode and reductions on depression and MCOP measures, these were attributed to small but significant reductions in the control group [CDI: $F(1,1078)=8.13, p<0.01, \eta^{2}=0.01$; and MCOP $\left.F(1,1078)=10.84, p<0.01, \eta^{2}=0.01\right]$, indicating changes in observed mean scores were not due to the intervention. The absence of any observed intervention effects meant that the prerequisite for testing hypotheses 1 and 3 was not met.

For the remaining structural equation hypothesis, H2, a Spearman's rank order correlation matrix was produced to confirm the presence of relationships between predictor and criterion variables, a prerequisite for testing mediation, and to examine presence of covariates (Table 3).

Socially prescribed perfectionism scores correlated moderately $(d=0.63)$ and positively with depression symptoms and scores for MCOP strategies, while correlations with scores on assistance seeking were negative and weaker. SOP scores positively correlated with all of the coping scores however did not correlate with depression. Adaptive coping correlated weakly and negatively with depression symptoms, while MCOP correlated positively with symptoms of depression. The mediation criteria of correlations between depression, SPP, and coping were satisfied.

Gender was correlated with more than two of the observed variables, each of which measured at least two different latent variables, thereby indicating that it was likely to influence interactions among the latent variables in the pathway model and needing to be controlled for in the SEM. Subsequently, a partial correlation matrix controlling for gender was computed and entered into LISREL. Incidentally, there were no significant differences in correlations between the Spearman and gender controlled correlation matrices.

\section{Hypothesis Testing}

The mediation hypothesis was tested by specifying pathways between latent variables (perfectionism, depression and coping) in LISREL to reflect the hypothesized relationships. In addition, measurement variables were specified for perfectionism (SOP and SPP), coping (maladaptive and adaptive), and depression (CDI). Measurement errors for subscales were derived from the Cronbach alpha rates from published studies and were entered into the SEM or computed by the model where no published data were available. Two competing structural models - a saturated model (Figure 1) with a maximum number of pathways and a nested, more parsimonious model testing mediation (Figure 2) - were then tested for fit and compared. Fit statistics for the two models are shown in Table 4.

A sandwich estimator was used to adjust the path coefficients' SEs to control for the potentially inflationary effects of intra-school dependencies on the Type I error rate (51). Intraschool correlation coefficients were calculated by means of a 2 (year groups) $\times 6$ (outcomes) ANOVA. Year groups accounted for $0.002-3.7 \%$ of variance on scores of output measures. $p$-Values for pathway coefficients were adjusted accordingly.

Path coefficients for predicted pathways were significant in both models with the exception of the SOP and MCOP path. Although the mediator model produced a reasonable fit, the statistics for the saturated model indicated a better fit. The fit statistics were significantly different with $\chi^{2} \mathrm{diff}=31.83, \mathrm{df}=2$, $p<0.001$, confirming that the saturated model produced the superior fit and contributed to $16.5 \%$ of the variance in depression scores at pre-test.

The direct pathways from SOP and SPP to depression (DEP) on the better fitting model were both significant. The model was therefore further examined to determine whether conditions were met for mediation, which is whether MCOP partially mediated these relationships. Conditions required to meet mediation are described below.

Condition 1: DEP needs to be correlated with both SOP and SPP. The correlation matrix of latent variables presented below indicates that this condition is satisfied (Table 5).

Condition 2: The three component pathways that comprise the indirect effects $(\mathrm{SOP} \rightarrow \mathrm{MCOP}, \quad \mathrm{SPP} \rightarrow \mathrm{MCOP}$, and $\mathrm{MCOP} \rightarrow \mathrm{DEP}$ ) must all be significant. The $p$-values reported in Figure 2 indicate that this condition is satisfied for only two of the three component pathways (SPP $\rightarrow$ MCOP and $\mathrm{MCOP} \rightarrow \mathrm{DEP})$.

Condition 3: The overall indirect effect from SPP to DEP via MCOP must be significant. The strength of the indirect effect is given by the product of its two component path coefficients; 0.39 multiplied by 0.19 equals -0.074 , which is significantly $>0$ $(z=3.10, p=0.002)$. The indirect effect from SPP to DEP via MCOP is therefore significant, which satisfies Condition 3. 
TABLE 3 | Spearman correlations of observed measures at pre-test.

\begin{tabular}{|c|c|c|c|c|c|c|c|c|c|}
\hline Variable & 1 & 2 & 3 & 4 & 5 & 6 & 7 & 8 & 9 \\
\hline 2. Gender & 0.07 & - & & & & & & & \\
\hline 4. Socially prescribed perfectionism & $-0.14^{\star \star}$ & $-0.14^{\star \star}$ & $0.30^{\star \star}$ & - & & & & & \\
\hline 5. Self-oriented perfectionism & $-0.10^{\star}$ & 0.04 & 0.03 & $0.44^{\star \star}$ & - & & & & \\
\hline 6. Adaptive coping-assistance seeking & 0.03 & $0.21^{\star \star}$ & $-0.12^{\star \star}$ & $-0.16^{\star \star}$ & $0.11^{*}$ & - & & & \\
\hline 7. Adaptive coping-cognitive behavioral problem solving & 0.03 & $0.13^{\star *}$ & $-0.10^{*}$ & 0.05 & $0.15^{\star \star}$ & $0.45^{\star \star}$ & - & & \\
\hline
\end{tabular}

$N=541$. ICSEA, index of community socio-educational advantage (54). ${ }^{*} p<0.05$, two-tailed; ${ }^{* *} p<0.01$, two-tailed.

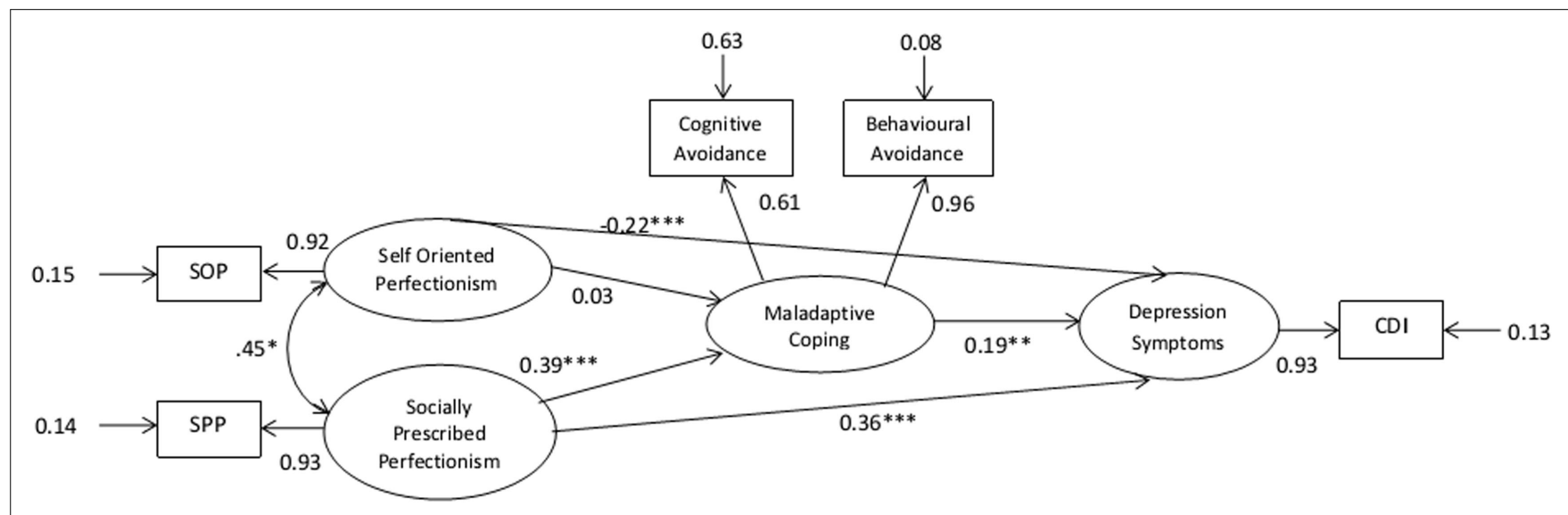

FIGURE 1 | Gender controlled saturated structural equation model at pre-test. $N=541 .{ }^{*} p<0.05$, two-tailed; ${ }^{* \star} p<0.01$, two-tailed; ${ }^{\star \star \star} p<0.001$.

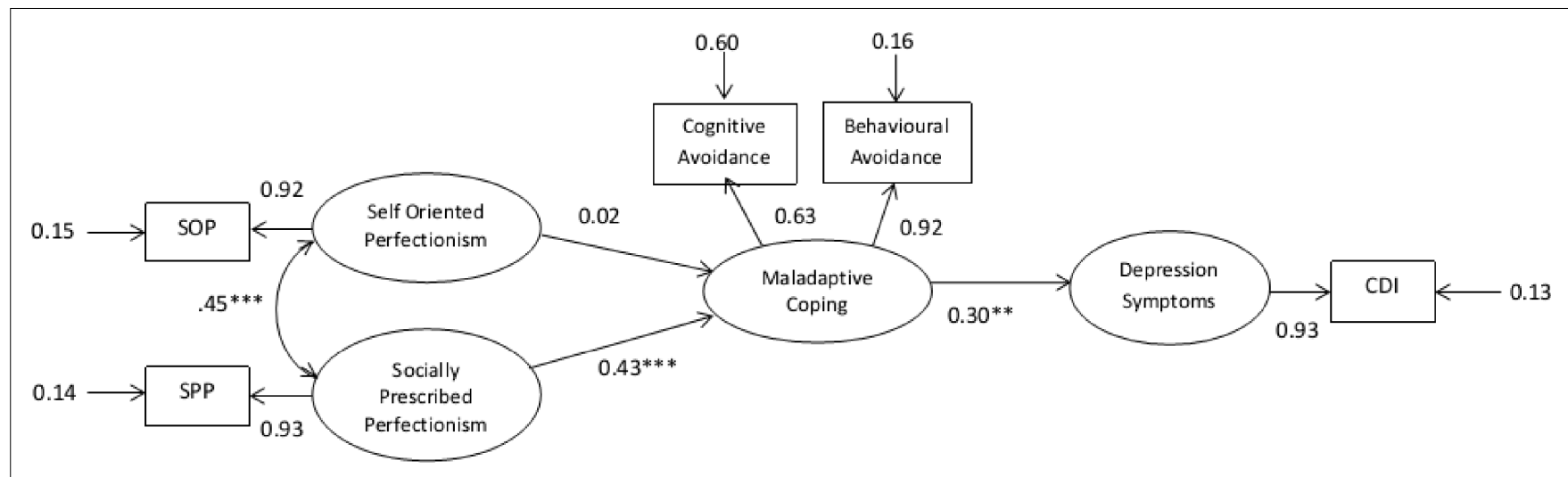

FIGURE 2 | Gender controlled mediator structural equation model at pre-test. $N=541 .{ }^{*} p<0.05$, two-tailed; ${ }^{\star \star} p<0.01$, two-tailed; ${ }^{* \star \star} p<0.001$.

TABLE 4 | Fit statistics for the measurement components of the saturated and mediator model.

\begin{tabular}{|c|c|c|c|c|c|}
\hline Model & $\chi^{2} / \mathrm{df}$ & $\begin{array}{l}\text { Comparative fit } \\
\text { index (CFI) }\end{array}$ & $\begin{array}{l}\text { Non-normed fit } \\
\text { index (NNFI) }\end{array}$ & $\begin{array}{l}\text { Standardized root mean square } \\
\text { residual (SRMR) }\end{array}$ & $\begin{array}{c}\text { Root mean square error of } \\
\text { approximation (RMSEA) }\end{array}$ \\
\hline Saturated model & $10.12 / 2=5.06$ & 0.98 & 0.92 & 0.02 & 0.13 (90\% Cl: $0.04,0.14)$ \\
\hline Mediator model & $41.95 / 4=10.49$ & 0.93 & 0.81 & 0.05 & 0.13 (90\% Cl: $0.09,0.16)$ \\
\hline
\end{tabular}


TABLE 5 | Correlation matrix of latent variables.

\begin{tabular}{lcccc}
\hline & SOP & SPP & MCOP & DEP \\
\hline SOP & 1 & & & \\
SPP & $0.525, p<0.001$ & 1 & 1 & \\
MCOP & $0.236, p<0.001$ & $0.408, p<0.001$ & & 1 \\
DEP & $0.008, p=0.871$ & $0.317, p<0.001$ & $0.282, p<0.001$ & \\
\hline
\end{tabular}

Condition 4: The strength of the direct pathway from SPP to DEP (0.36) must be significantly less than the strength of this pathway after removing MCOP from the model (0.43). A $z$ value of 1.96 or greater is required for significance at the 0.05 significance level. However, the computed $z$ value was 0.84 and therefore suggests that there is no significant difference between the two path coefficients. Unfortunately, the fourth condition for partial mediation is not satisfied.

To conclude, SPP had both a direct and indirect effect (via mcop) on depression. The indirect effect, however, does not reflect partial mediation.

\section{Discussion}

The research aims of this study were to examine whether perfectionism limited the effectiveness of an intervention aimed at preventing depression in preadolescents, whether coping mediated the relationship between perfectionism and depression at pre-test, and whether increases in coping facilitated the effectiveness of the intervention.

The absence of an observed intervention effect rendered hypothesis 1 and 3 untestable. Considering that a previous trial of the AO-PTS intervention found significant effects in a larger sample of children where mean CDI scores were approximately 1 SD higher $(M=12.21, N=467)$ than those of the current sample, a key factor limiting the observation of an intervention effect in this sample was an apparent floor effect of scores for depression symptoms, potentially exacerbated by wave attrition of a disproportionate number of children with elevated scores for depression in the control group. Additionally, as children with elevated scores for depression were offered referrals for psychological assistance, any external support received may have contributed to the observed reduction in depression scores. It is possible that this contributed to the observed pre-post-test reductions in the control group, which comprised a higher number of at-risk children who may have received psychological help, thereby mitigating any treatment effect. Additional factors limiting observation of an intervention effect included potential temporal effects due to differences in timing of testing for control and intervention groups and potential inconsistencies in treatment compliance by teachers (this could not be determined due to incomplete records of treatment fidelity and child attendance rates for some schools).

It was predicted that at pre-test, MCOP would partially mediate the relationship between perfectionism and depression symptoms and that this mediation relationship would be stronger between depression and SPP. Results confirmed that gender was a covariate, influencing several of the outcome measures. The SEM found that SPP had a direct and indirect association with depression but that coping was not a mediator. This finding suggests that while children with high levels of SPP tend to employ more dysfunctional coping strategies to manage stress, thereby contributing to depression symptoms, other perfectionistic tendencies, such as self-criticism and rumination, over failure to meet others' perceived expectations have an independent and stronger relationship with depression. These findings are contrary to SEM research in adults, which demonstrated more clearly that avoidant coping mediated the relationship between perfectionism and depression [e.g., Ref. $(30,52)]$. Wei et al's (30) more sophisticated longitudinal design demonstrated that maladaptive perfectionism and coping not only contributed to depression but also influenced each other at different points in time. Dunkley and Blankstein's (52) mediation model was demonstrated in the context of daily hassles and distress. It is feasible that the more complex models of coping and perfectionism tapped into the latent variables more effectively. Zhang and Cai (31) claimed partial mediation in a simpler cross-sectional model; however, the patterns of association observed in their sample of young adults were similar to those observed in this sample with direct pathways relatively stronger compared to indirect pathways, casting doubt over their claim of partial mediation.

The SEM demonstrated a small but significant negative association between depression and SOP, indicating that there may be protective factors associated with this dimension of perfectionism. SOP also correlated positively with cognitive behavioral problem solving, indicating that children who are more self-motivated to achieve may be less likely to suffer from depression. However, this conclusion would be inconsistent with findings in adult and child literature by Nobel et al. who found a positive association between SOP and depression. This contradiction may be due to differences in levels of distress in the sample, with Nobel et al.s sample showing higher levels of depression symptoms. Hewitt et al. (16) and O'Connor et al. (17) observed that stress moderated the association between perfectionism (including SOP) and depression in adolescent youth. This would suggest that maladaptive cognitions associated with SOP, such as rumination over mistakes, procrastination, and self-criticism, may only be triggered in the presence of acute stress. Lewis and Frydenberg (25) observed that adolescents who implemented adaptive coping strategies also used MCOP strategies, concluding that adolescents may adopt MCOP strategies when other strategies have failed. Others have suggested that avoidance strategies may be the default choice in times of distress $(25,44)$.

Consistent with research by Flett et al. (28), SPP scores showed a moderate and positive association with MCOP, while SPP was negatively associated with adaptive coping, suggesting that children of this younger age group with tendencies to critically evaluate themselves against perceived high expectations tend to use more behavioral and cognitive avoidance strategies to deal with stressors.

\section{Strengths and Limitations}

The strengths of this study included a sound methodological approach comprising a randomized control design, standardized testing procedures, a large sample size ensuring a sufficiently powered statistical analysis, and sophisticated statistical methods to assess the interaction of outcome variables, which were able to 
account for measurement error, group inequivalence, and intraclass dependence.

Because the mediation hypothesis was tested cross-sectionally, inferences regarding causal relationships implied in the model are limited as the temporal sequencing of perfectionism, coping, and depression was not demonstrated (53). Ideally, mediation is tested longitudinally over three points in time without potential interference of an intervention. As data were unavailable for three separate time points, it was decided to examine meditational interactions concurrently at pre-test, combining intervention and control subjects to maximize sample size and thereby increasing statistical power. Other limitations included reliance on self-reports of children and poor reliability rates on two of the subscales of the CSCY, which may have limited observed relationships (although error rates were accounted for in the SEM). Finally, the generalizability of results is limited as participating children were from predominantly Catholic middle income families.

\section{Practical and Theoretical Implications}

This study extended on a very limited body of research demonstrating links between MCOP and perfectionism and presenting novel research results of SEM of the interrelationships between socially prescribed and SOP, coping, and depression. The pathway model identified provides an insight into the interactions of these variables in asymptomatic children, and also provides a more accurate analysis (by accounting for measurement error) of relationships between perfectionism and depression, which has previously been examined primarily through means of correlational studies.

It is recommended that future research examine these interrelationships in a longitudinal study to demonstrate temporal sequence in a causal model. It is also advised that a coping measure is used, which has demonstrated strong internal consistency across all subscales to improve construct validity. Further research validating the CSYC for use in a younger sample should be considered. Future examination of the relationship between SOP

\section{References}

1. Bergman AJ, Nyland JE, Burns LR. Correlates with perfectionism and the utility of a dual process model. Pers Individ Dif (2007) 43(2):389-99. doi:10.1016/j. paid.2006.12.007

2. Bieling PJ, Israeli AL, Antony MM. Is perfectionism good, bad, or both? Examining models of the perfectionism construct. Pers Individ Dif (2004) 36(6):1373-85. doi:10.1016/s0191-8869(03)00235-6

3. Flett GL, Hewitt PL. Perfectionism and maladjustment: an overview of theoretical, definitional, and treatment issues. In: Flett GL, Hewitt PL, editors. Perfectionism: Theory, Research and Treatment. Washington, DC: American Psychological Association (2002). p. 5-27.

4. Owens RG, Slade P. So perfect it's positively harmful? Reflections on the adaptiveness and maladaptiveness of positive and negative perfectionism. Behav Modif (2008) 32(6):928-37. doi:10.1177/0145445508319667

5. Frost RO, Martin P, Lahart C, Rosenblate R. The dimensions of perfectionism. Cognit Ther Res (1990) 14:449-68. doi:10.1007/BF01172967

6. Terryshort LA, Owens RG, Slade PD, Dewey ME. Positive and negative perfectionism. Pers Individ Dif (1995) 18(5):663-8. doi:10.1016/0191-8869(94) 00192-U

7. Frost RO, Trepanier KL, Brown EJ, Heimberg RG, Juster HR, Makris GS, et al. Self-monitoring of mistakes among subjects high and low in perfectionistic concern over mistakes. Cognit Ther Res (1997) 21:209-22. doi:10.1023/A: 1021884713550 coping and depression in a diathesis-stress model may highlight maladaptive traits of SOP in healthy samples, which could further inform the formulation of preventive strategies. As suggested by Pincus and Friedman (26), such preventative interventions may need to include not only an increase in adaptive coping skills but also an increased awareness of the effects of using less productive coping strategies in times of stress. Future research testing for moderation of perfectionism on efficacious treatments in children is warranted as is an investigation into whether improving coping skills would buffer against the effects of perfectionism on depression.

\section{Conclusion}

The study through sophisticated SEM supported that children with a tendency to evaluate themselves critically against the perceived unrealistically high expectations of others tend to report higher levels of depression. This influence is predominantly due to the maladaptive cognitions associated with perfectionism, and to a lesser extent, as a result of an increased tendency of these children toward to use of ineffective behavioral and cognitive avoidance strategies to cope with stressors.

Furthermore, the findings that associations between perfectionism, MCOP, and depression are present even in children who do not necessarily present clinical levels of depression symptoms could be useful in informing universal programs to prevent adverse mental health outcomes in children.

\section{Acknowledgments}

We thank Dr. Melissa C. Davis for her support and contributions in the early stages of this project. We also acknowledge the significant contribution of the teachers who facilitated the program, and the students and families that participated in the research. The study was supported by grants from Healthway and Curtin University's Division of Health Sciences.

8. Shafran R, Mansell W. Perfectionism and psychopathology: a review of research and treatment. Clin Psychol Rev (2001) 21:879-906. doi:10.1016/ s0272-7358(00)00072-6

9. Bieling PJ, Summerfeldt LJ, Israeli AL, Antony MM. Perfectionism as an explanatory construct in comorbidity of axis I disorders. J Psychopathol Behav Assess (2004) 26(3):193-201. doi:10.1023/B:JOBA.0000022112.27186.98

10. Egan SJ, Wade TD, Shafran R. Perfectionism as a transdiagnostic process: a clinical review. Clin Psychol Rev (2011) 31:203-12. doi:10.1016/j.cpr.2010.04.009

11. Kovacs M, Lopez-Duran N. Prodromal symptoms and atypical affectivity as predictors of major depression in juveniles: implications for prevention. J Child Psychol Psychiatry (2010) 51(4):472-96. doi:10.1111/j.1469-7610.2010. 02230.x

12. Costello EJ, Mustillo S, Erkanli A, Keeler G, Angold A. Prevalence and development of psychiatric disorders in childhood and adolescence. Arch Gen Psychiatry (2003) 60:837-44. doi:10.1001/archpsyc.60.8.837

13. Hewitt PL, Flett GL. Perfectionism in the self and social contexts: conceptualization, assessment, and association with psychopathology. J Pers Soc Psychol (1991) 60(3):456-70. doi:10.1037/0022-3514.60.3.456

14. Flett GL, Coulter L-M, Hewitt PL, Nepon T. Perfectionism, rumination, worry, and depressive symptoms in early adolescents. Can J Sch Psychol (2011) 26:159-76. doi:10.1177/0829573511422039

15. Fuhr K, Hautzinger M, Meyer TD. Implicit motives and cognitive variables: specific links to vulnerability for unipolar or bipolar disorder. Psychiatry Res (2013) 215:61-8. doi:10.1016/j.psychres.2013.10.001 
16. Hewitt PL, Caelian CF, Flett GL, Sherry SB, Collins L, Flynn CA. Perfectionism in children: associations with depression, anxiety, and anger. Pers Individ Dif (2002) 32:1049-61. doi:10.1016/S0191-8869(01)00109-X

17. O'Connor RC, Rasmussen S, Hawton K. Predicting depression, anxiety and selfharm in adolescents: the role of perfectionism and acute life stress. Behav Res Ther (2010) 48:52-9. doi:10.1016/j.brat.2009.09.008

18. Nobel R, Manassis K, Wilansky-Traynor P. The role of perfectionism in relation to an intervention to reduce anxious and depressive symptoms in children. J Ration Emot Cogn Behav Ther (2011) 30(2):1-14. doi:10.1007/s10942-0110133-5

19. Huggins L, Davis MC, Rooney R, Kane R. Socially prescribed and self-oriented perfectionism as predictors of depressive diagnosis in preadolescents. Aus J Guid Couns (2008) 18:182-94. doi:10.1375/ajgc.18.2.182

20. Aldea MA, Rice KG. The role of emotional dysregulation in perfectionism and psychological distress. J Couns Psychol (2006) 53(4):498-510. doi:10.1037/ 0022-0167.53.4.498

21. Dunkley DM, Zuroff DC, Blankstein KR. Self-critical perfectionism and daily affect: dispositional and situational influences on stress and coping. J Pers Soc Psychol (2003) 84(1):234-52. doi:10.1037/0022-3514.84.1.234

22. Aldao A, Nolen-Hoeksema S, Schweizer S. Emotion-regulation strategies across psychopathology: a meta-analytic review. Clin Psychol Rev (2010) 30(2):217-37. doi:10.1016/j.cpr.2009.11.004

23. Compas BE, Connor-Smith JK, Saltzman H, Thomsen AH, Wadsworth ME. Coping with stress during childhood and adolescence: problems, progress, and potential in theory and research. Psychol Bull (2001) 127(1):87-127. doi:10. 1037/0033-2909.127.1.87

24. Holen S, Lervåg A, Waaktaar T, Ystgaard M. Exploring the associations between coping patterns for everyday stressors and mental health in young schoolchildren. J Sch Psychol (2012) 50(2):167-93. doi:10.1016/j.jsp.2011.10.006

25. Lewis R, Frydenberg E. Adolescents least able to cope: how do they respond to their stresses? Br J Guid Counc (2004) 32(1):25-37. doi:10.1080/ 03069880310001648094

26. Pincus DB, Friedman AG. Improving children's coping with everyday stress: transporting treatment interventions to the school setting. Clin Child Fam Psychol Rev (2004) 7(4):223-40. doi:10.1007/s10567-004-6087-8

27. Rudolph S, Flett G, Hewitt P. Perfectionism and deficits in cognitive emotion regulation. J Ration Emot Cogn Behav Ther (2007) 25(4):343-57. doi:10.1007/ s10942-007-0056-3

28. Flett GL, Druckman T, Hewitt PL, Wekerle C. Perfectionism, coping, social support, and depression in maltreated adolescents. J Ration Emot Cogn Behav Ther (2012) 30(2):118-31. doi:10.1007/s10942-011-0132-6

29. Dunkley DM, Sanislow CA, Grilo CM, McGlashan TH. Perfectionism and depressive symptoms 3 years later: negative social interactions, avoidant coping, and perceived social support as mediators. Compr Psychiatry (2006) 47(2):106-15. doi:10.1016/j.comppsych.2005.06.003

30. Wei M, Heppner PP, Russell DW, Young SK. Maladaptive perfectionism and ineffective coping as mediators between attachment and future depression: a prospective analysis. J Couns Psychol (2006) 53(1):67. doi:10.1037/0022-0167. 53.1.67

31. Zhang B, Cai T. Coping styles and self-esteem as mediators of the perfectionism-depression relationship among Chinese undergraduates. Soc Behav Pers (2012) 40(1):157. doi:10.2224/sbp.2012.40.1.157

32. Blatt SJ. The destructiveness of perfectionism - implications for the treatment of depression. Am Psychol (1995) 50:1003-20. doi:10.1037/0003-066X.50.12. 1003

33. Arpin-Cribbie C, Irvine J, Ritvo P, Cribbie RA, Flett GL, Hewitt PL. Perfectionism and psychological distress: a modeling approach to understanding their therapeutic relationship. J Ration Emot Cogn Behav Ther (2008) 26(3):151-67. doi:10.1007/s10942-007-0065-2

34. Egan SJ, Hine P. Cognitive behavioural treatment of perfectionism: a single case experimental design series. Behav Change (2008) 25:245-58. doi:10.1375/bech. 25.4.245

35. Riley C, Lee M, Cooper Z, Fairburn CG, Shafran R. A randomised controlled trial of cognitive-behaviour therapy for clinical perfectionism: a preliminary study. Behav Res Ther (2007) 45:2221-31. doi:10.1016/j.brat.2006.12.003
36. Essau CA, Conradt J, Sasagawa S, Ollendick TH. Prevention of anxiety symptoms in children: results from a universal school-based trial. Behav Ther (2012) 43(2):450-64. doi:10.1016/j.beth.2011.08.003

37. Dry SM. Moderation Effect of Perfectionism on Changes in Internalising Symptoms in preadolescents (Unpublished honours dissertation) School of Psychology and Speech Pathology. Perth, WA: Curtin University (2011).

38. Park H-J, Heppner PP, Lee D-G. Maladaptive coping and self-esteem as mediators between perfectionism and psychological distress. Pers Individ Dif (2010) 48(4):469-74. doi:10.1016/j.paid.2009.11.024

39. Kline RB. Principles and Practice of Structural Equation Modeling. 3rd ed. New York, NY: The Guildford Press (2011).

40. Rooney R, Rudge L, Snowball L, Roberts CM, Pike L, Mullan A, et al. The Positive Thinking Program: Prevention Manual. Perth, WA: Curtin University of Technology (2004).

41. Rooney R, Hassan S, Kane R, Roberts CM, Nesa M. Prevention and reduction of internalising disorders in 9-10 year old children: a universal randomized controlled trial. Behav Res Ther (2013) 51:845-54. doi:10.1016/j.brat.2012.09.005

42. Kovacs M. Children's Depression Inventory Manual. New York, NY: MultiHealth System Inc (1992).

43. Rooney R, Roberts CM, Kane R, Pike L, Winsor A, White J, et al. The prevention of depression in 8- to 9-year-old children: a pilot study. Aus J Guid Couns (2006) 16:76-90. doi:10.1375/ajgc.16.1.76

44. Brodzinsky DM, Elias MJ, Steiger C, Simon J, Gill M, Hitt JC. Coping scale for children and youth: scale development and validation. J Appl Dev Psychol (1992) 13(2):195-214. doi:10.1016/0193-3973(92)90029-h

45. Jöreskog KG, Sörbom D. LISREL 8.80 for Windows [Computer Software]. Lincoln, IL: Scientific Software International Inc (2006).

46. Tabachnick BG, Fidell LS. Using Multivariate Statistics. 5th ed. Boston, MA: Pearson/Allyn \& Bacon (2007).

47. Collins LM, Schafer JL, Kam CM. A comparison of inclusive and restrictive missing-data strategies in modern missing-data procedures. Psychol Methods (2001) 6:330-51. doi:10.1037/1082-989X.6.4.330

48. Schafer JL, Graham JW. Missing data: our view of the state of the art. Psychol Methods (2002) 7:147-77. doi:10.1037//1082-989X.7.2.147

49. Coakes SJ, Steed L, Ong C. SPSS: Analysis Without Anguish Using SPSS Version 17.0 for Windows. Milton, QLD: John Wiley \& Sons Australia Ltd (2009).

50. Holden JE. Analyzing change: a primer on multilevel models with applications to nephrology. Am J Nephrol (2008) 28(5):792-801. doi:10.1159/ 000131102

51. Rabe-Hesketh S, Skrondal A. Multilevel and Longitudinal Modelling Using Stata. College Station, TX: StataCorp (2005).

52. Dunkley DM, Blankstein KR. Self-critical perfectionism, coping, hassles, and current distress: a structural equation modeling approach. Cognit Ther Res (2000) 24(6):713-30. doi:10.1023/a:1005543529245

53. Shadish WR, Cook TD, Campbell DT. Experimental and Quasi-Experimental Designs for Generalized Causal Inference. Boston, MA: Houghton Mifflin (2001).

54. Australian Curriculum Assessment and Reporting Authority. Index of Community Socio-Educational Advantage (2012). Available from: http://myschool. edu.au/

55. Hu L, Bentler PM. Cutoff criteria for fit indexes in covariance structure analysis: conventional criteria versus new alternatives. Struct Equation Model (1999) 6:1-55. doi:10.1080/10705519909540118

56. Jaccard J, Wan CK. LISREL Analyses of Interaction Effects in Multiple Regression. Newbury Park, CA: SAGE (1996).

Conflict of Interest Statement: The authors declare that the research was conducted in the absence of any commercial or financial relationships that could be construed as a potential conflict of interest.

Copyright (c) 2015 Dry, Kane and Rooney. This is an open-access article distributed under the terms of the Creative Commons Attribution License (CC BY). The use, distribution or reproduction in other forums is permitted, provided the original author(s) or licensor are credited and that the original publication in this journal is cited, in accordance with accepted academic practice. No use, distribution or reproduction is permitted which does not comply with these terms. 